\title{
Fair Resource Allocation under Rayleigh and/or Rician Fading Environments
}

\author{
Erwu Liu and Kin K. Leung \\ Department of Electrical and Electronic Engineering \\ Imperial College \\ London, United Kingdom \\ \{erwu.liu,kin.leung@imperial.ac.uk\}
}

\begin{abstract}
Proportional fair scheduling (PFS) provides good balance between throughput and fairness via multi-user diversity and game-theoretic equilibrium. Very little analytical work exists on understanding the performance of PFS. Most existing prior results are for networks with Rayleigh fading. In this paper, we provide theoretical results for PFS in general fading environments. The results reveal that the average throughput of a user solely depends on its own channel statistics when its instantaneous data rate is Gaussian. Based on the theoretical results, we analyze the $P F S$ performance under various scenarios with Rayleigh and/or Rician fading, and the numerical results match very well with the simulation ones. To the best of our knowledge, this work is the first one theoretically investigating the PFS problem in general fading environments.
\end{abstract}

Keywords- proportional fair scheduling, multi-user diversity, Gaussian, Rayleigh fading, Rician fading

\section{INTRODUCTION}

In the considerable efforts to deal with the tradeoff between optimal system throughput and user fairness, the proportional fair scheduling $(P F S)$ algorithm is proposed. Since its presence [1], there has been substantial interest in the PFS algorithm in wireless networks (see [2,3] and the references therein).

Though the PFS algorithm has got so much attention and currently implemented in $3 \mathrm{G}$ wireless network for high data rate delay-tolerant services [4], one can only see very limited analysis results on PFS [5-11], whose derivations typically assume a simple linear rate model or logarithm rate model and/or use a simplified form of the original PFS preference metric. The linear rate model is only valid for networks where the signal to interference-plus-noise ratio $(S I N R)$ is very small [5], while the logarithm rate model can only be used for singleinput-single-output (SISO) communications [8]. The use of linear rate model or logarithm rate model is a reasonable modeling convention. However, when examining throughput performance, it does not seem satisfactory to assume such simplified rate models. Moreover, all existing researches are assuming some kind of independent identically distributed (i.i.d) relationship among users in their derivations [5-9, 11]. Undoubtedly, these assumptions limit the use of existing theoretical results on PFS.

Our previous work [12] provides an analytic expression for the throughput of $P F S$ in such a scenario where the instantaneous data rate over a Rayleigh fading channel can be approximated by a Gaussian distribution [13, 14]. Different from the linear rate model and logarithm rate model, the Gaussian approximation (GA) method used in [12] applies to both low and high SINR cases, moreover, it can be used to accurately model the instantaneous data rate of a multipleinput-multiple-output (MIMO) link over a Rayleigh/Rician fading channel. The main drawback of our prior work is that it assumes a proportional relationship between the mean and standard deviation of instantaneous data rate in the derivation.

While most existing results are for Rayleigh fading networks, we would like to point out that a realistic network typically has fundamentally different fading processes in practice. This paper extends our prior work to such general fading environments. Specifically, we study the PFS problem in 4 different scenarios: Gaussian rate network, Rayleigh fading network, Rician fading, and Rayleigh+Rician hybrid fading network without the mathematic simplifications above.

The rest of the paper is structured as follows. Related work is discussed in Section II. After that, analytical results which extend our prior work on PFS are presented in Section III. Finally, in Section IV, we present simulation results to validate the theoretical findings under various fading environments, followed by the conclusion in Section V.

\section{RELATED WORK}

Since its first presence in Kelly's seminal paper [1], significant efforts has been put into the study of the PFS algorithm. Holtzman [5] conducted the asymptotic analysis of the PFS algorithm, with a result that all other things being equal, the user class with more fading variability gets more throughput. Kushner et al. [7] investigated the convergence property of the algorithm. They stated that the limiting behavior of the throughputs converges to the solution of an ordinary differential equation, and found the limit throughput is proportional to the average instantaneous rate for Rayleigh fading by assuming the instantaneous rate is proportional to the received SINR which is i.i.d for all users. Also, [11] presented some results on PFS for the scenario where the relative rate fluctuations are statistically identical, stating that each user would receive same amount of the time slots.

To simplify the problem, most existing analytic results are assuming some kind of i.i.d relationship among users [5-9, 11]. For example, [6, 7] assume the SINR of each user is an i.i.d Exponential distribution; [8] takes the assumption similar to [6] that user $k$ 's SINR $S_{k}=c_{k} \times C(\forall k)$, where $c_{k}$ is a user-related 
constant and $C$ is an i.i.d Exponential distribution random variable independent of all users, and [11] assumes that the instantaneous data rate of user $i$ with time-average rate $C_{i}$ is distributed as $R_{i}=C_{i} Y_{i} Z$, where $Y, Y_{1}, Y_{2}, \ldots$ are i.i.d copies and $Z$ represent a possible correlation component with unit mean, and the exponentially smoothed throughput of user $i$ scale linearly with the time-average rate $C_{i}$, i.e., $W_{i}=C_{i} V_{i}$, where the random variables $V_{1}, \ldots, V_{\mathrm{m}}$ are identically distributed.

On the other hand, linear rate model and logarithm rate model are the two rate models commonly used for analyzing the performance of PFS. For example, [5, 6, 11] use the linear rate model, while [8] uses the logarithm rate model. In the linear rate model, the instantaneous data rate $R$ of a user is linearly proportional to the received SINR, i.e, $R_{i}=\beta \times S I N R_{i}$ for any user $i$; while in the logarithm rate model, there is a logarithmic relationship between the received SINR and the instantaneous data rate, i.e., $R_{i} \propto \log _{2}\left(1+\beta \times S I N R_{i}\right)$. These two rate models have their merits as they greatly simplify the mathematical analysis of PFS. However, such simplified models may not be satisfactory enough to characterize the instantaneous data rate in practical network environments, especially where there are fundamentally different fading processes. As stated in [5], the linear rate model is usually a reasonable approximation for small SINR and is not accurate when multiple modulations or codings are used. On the other hand, the logarithm rate model can only be used for SISO links and is a very rough approximation when used for MIMO links [8]. In fact, works by Telatar [13] and Smith et al. [14] suggested that the instantaneous rate over Rayleigh or Rician fading channels can be better modeled by a Gaussian distribution with surprisingly high accuracy. Thus the Gaussian approximation $(G A)$ instead of the two simplified rate models is used in our prior research to study the $P F S$ performance [12]. The main drawback of [12] is that it assumes a proportional relationship between the mean and standard deviation of instantaneous data rate.

It is not surprising that most studies mentioned above are for Rayleigh fading networks as a system with more fading variability will get more $P F S$ benefits [5]. In fact, the channel characteristics in a realistic network should be better modeled as a mixture of Rayleigh and Rician fading. This paper will also consider such fading scenarios.

In all, our goal is to study the PFS performance in general fading wireless networks without the mathematic simplifications used in existing researches.

\section{PFS: THEORETICAL RESULTS}

We first outline our previous results. Then we present the analytical expression of PFS in general fading environments.

In [12], we have the following important result:

In an N-user cellular network implementing the PFS algorithm, the average throughput of user $j$ satisfies

$$
E\left[\mu_{j}\right]=\int_{0}^{\infty} x f_{R_{j}}(x) \operatorname{Pr}\left(I_{j}=1 \mid R_{j}=x\right) d x
$$

where $\operatorname{Pr}\left(I_{j}=1 \mid R_{j}=x\right)$ is the conditional probability of user $j$ to be scheduled given its instantaneous data rate $R_{j}$,

$$
\operatorname{Pr}\left(I_{j}=1 \mid R_{j}=x\right)=\prod_{i=1, i \neq j}^{N} F_{R_{i}}\left(\frac{E\left[\mu_{i}\right]}{E\left[\mu_{j}\right]} x\right)
$$

$f_{R i}(),. F_{R i}($.$) are the probability density function (pdf) and$ cumulative distribution function (cdf) of the instantaneous data rate of user $i, \mu_{i}$ is the throughput of user $i, E[$.$] is the$ statistical average, and $I_{j}$ is the indicator function of the event that user $j$ is scheduled to transmit in next time slot,

$$
I_{j}=\left\{\begin{array}{l}
1, j \text { is scheduled to transmit in next time slot } \\
0, \text { else }
\end{array}\right.
$$

Simulations (Figure 4 and 5) have found that, in Rayleigh, Rician or Rayleigh+Rician hybrid fading environments, $E[\mu] / E[R]$ is a monotonically decreasing function of $E[R]$, while $E[\mu] / \sigma_{R}$ is a monotonically increasing function of $\sigma_{R}$, i.e., $\sigma_{R i} / \sigma_{R j} \leq E\left[\mu_{i}\right] / E\left[\mu_{j}\right] \leq E\left[R_{i}\right] / E\left[R_{j}\right]$ given $0 \leq E\left[R_{j}\right] \leq E\left[R_{i}\right]$. Denote $E\left[R_{j}\right] / \sigma_{R j}$ by $M_{j}$, we have

$$
\begin{aligned}
E\left[\mu_{j}\right] \leq & \sigma_{R_{j}} \int_{-M_{j}}^{\infty}\left(y \sigma_{R_{j}}+E\left[R_{j}\right]\right) f_{R_{j}}\left(y \sigma_{R_{j}}+E\left[R_{j}\right]\right) \\
& \times\left[\prod_{\forall i \neq j, E\left[R_{i}\right] \geq E\left[R_{j}\right]}^{N} F_{R_{i}}\left(y \frac{E\left[R_{i}\right]}{E\left[R_{j}\right]} \sigma_{R_{j}}+E\left[R_{i}\right]\right)\right] \\
& \times\left[\prod_{\forall i \neq j, E\left[R_{i}\right] \leqslant E\left[R_{j}\right]}^{N} F_{R_{i}}\left(y \sigma_{R_{i}}+\frac{\sigma_{R_{i}}}{\sigma_{R_{j}}} E\left[R_{j}\right]\right)\right] d y
\end{aligned}
$$

and

$$
\begin{aligned}
E\left[\mu_{j}\right] \geq \sigma_{R_{j}} \int_{-M_{j}}^{\infty}\left(y \sigma_{R_{j}}+E\left[R_{j}\right]\right) f_{R_{j}}\left(y \sigma_{R_{j}}+E\left[R_{j}\right]\right) \\
\times\left[\prod_{\forall i \neq j, E\left[R_{i}\right] \geq E\left[R_{j}\right]}^{N} F_{R_{i}}\left(y \sigma_{R_{i}}+\frac{\sigma_{R_{i}}}{\sigma_{R_{j}}} E\left[R_{j}\right]\right)\right] \\
\times\left[\prod_{\forall i \neq j, E\left[R_{i} k E\left[R_{j}\right]\right.}^{N} F_{R_{i}}\left(y \frac{E\left[R_{i}\right]}{E\left[R_{j}\right]} \sigma_{R_{j}}+E\left[R_{i}\right]\right)\right] d y
\end{aligned}
$$

If we write

$$
\begin{aligned}
E\left[\mu_{j}\right] & \approx \sigma_{R_{j}} \int_{-M_{j}}^{\infty}\left(y \sigma_{R_{j}}+E\left[R_{j}\right]\right) f_{R_{j}}\left(y \sigma_{R_{j}}+E\left[R_{j}\right]\right) \\
& \times \prod_{\forall i=1, i \neq j}^{N} F_{R_{i}}\left(y \sigma_{R_{i}}+E\left[R_{i}\right]\right) d y
\end{aligned}
$$

It is easy to prove that the average throughput given by (6) lies between the upper and lower bounds given by (4) and (5), respectively.

Now we have the closed-form expressions (4)-(6) for the PFS throughput.

It is easy to prove that with $G A$ method for Rayleigh and/or Rician fading environments, (6) further reduces to

$$
\begin{aligned}
E\left[\mu_{j}\right] \approx & \frac{E\left[R_{j}\right\rfloor}{N} \times\left(1-\left[F_{(0,1)}\left(-E\left[R_{j}\right] / \sigma_{R_{j}}\right)\right]^{N}\right) \\
& +\int_{-M_{j}}^{\infty} y \sigma_{R_{j}} f_{(0,1)}(y) \times\left[F_{(0,1)}(y)\right]^{N-1} d y
\end{aligned}
$$

Moreover, if user $j$ 's instantaneous data rate $R_{j}=c_{j} \times S I N R$ $(\forall j)$, where $c_{j}$ is a user-related, positive constant and SINR is an 
i.i.d exponentially distributed random variable independent of all users, (6) can be written as.

$$
\begin{aligned}
E\left[\mu_{j}\right] & =\int_{0}^{\infty} c_{j} y \times f_{C}(y) \times\left(F_{C}(y)\right)^{N-1} d y \\
& =\int_{0}^{\infty} c_{j} y \times e^{-y} \times\left(1-e^{-y}\right)^{N-1} d y=c_{j} \times \frac{1}{N} \times \sum_{k=1}^{N} \frac{1}{k}
\end{aligned}
$$

In fact, (8) is the analytical result given by [6] and [8] for a Rayleigh fading network using the linear rate model.

Formula (7) has the great practical and theoretical interests of being mathematically graceful and simple.

(7) reveals a very interesting merit of PFS: node j's throughput is solely (approximately, if we ignore the effect of $M_{j}$ on the integral) determined by its own channel statistics when the instantaneous data rate is Gaussian.

Other observations can also be made from (7): Firstly, as $E\left[R_{j}\right] / N$ is the mean throughput of node $j$ when using round robin $(R R)$ scheduling, the second item in the right hand of (7) is in fact the improvement of node $j$ 's throughput when using PFS instead of RR scheduling. Secondly, PFS benefits more in severe fading networks where $\sigma_{R j}$ is large. On the other hand, $P F S$ can be viewed as a $R R$ scheduling algorithm in low-fading environments where $\sigma_{R j}$ is relatively small compared with $E\left[R_{j}\right]$. Similar observations have been made by others $[5,6,11]$.

We now conduct simulations for various fading scenarios to validate our theoretical results.

\section{Numerical ReSUlts AND SimUlation}

Throughout, we assume that users have independent fading channels.

We run the simulations for the following scenarios:

I the instantaneous data rate is Gaussian

II Rayleigh fading channel, MIMO link;

III Rayleigh fading channel, SISO link;

IV Rician fading channel, SISO link, and

V Rayleigh+Rician hybrid fading channel, SISO link.

Simulation parameters are: 4000 scheduling slots, moving average factor $k=500$. For scenarios other than case I, the instantaneous data rate (in bps/Hz) is given by $\log _{2}(1+\operatorname{SINR})$, where SINR is a random variable characterized by corresponding fading process.

Case I: Instantaneous data rate is Gaussian. Network size varies from 5 to 180 users. For each user, the instantaneous data rate $R$ is a Gaussian distribution with a mean $E[R]$ uniformly distributed between $1 \sim 8 \mathrm{bps} / \mathrm{Hz}^{*}$, and a standard deviation $\sigma_{R}$ uniform distributed between $0 \sim 0.4 E[R]^{* 2}$.

\footnotetext{
${ }^{* 1}$ A data rate within $1 \sim 8 \mathrm{bps} / \mathrm{Hz}$ corresponds to a practical modulation type (BPSK 256QAM).

${ }^{* 2}$ Assumed Gaussian, $R$ could be negative. So we set $\sigma_{R} \leq 0.4 E[R]$ to ensure the probability $\operatorname{Pr}\{R<0\} \approx 0.6 \%$.
}

Case II: Rayleigh fading network of 10 users with average $\operatorname{SINR}=1,2,3,4,5,6,7,8,9,10 d B$, respectively. $5 \times 5 \mathrm{MIMO}$ is used for the communication between a user and the access point.

Case III: Rayleigh fading network of 10 users with average $\operatorname{SIN} R=1,2,3,4,5,6,7,8,9,10 d B$, respectively.

Case IV: Rician fading: 10 users with average $\operatorname{SINR}=11,12,13,14,15,16,17,18,19,20 \mathrm{~dB}$, respectively.

Case V: Rayleigh+Rician hybrid fading: 10 Rayleigh fading users with average $\operatorname{SINR}=1,2,3,4,5,6,7,8,9,10 d B$, respectively, plus 10 Rician fading users with average $\operatorname{SINR}=11,12,13,14,15,16,17,18,19,20 d B^{* 3}$.

For cases III-V, SISO is used for the communication between a user and the access point (i.e., base station in cellular networks).

For the simplicity of presentation, each node is numbered by its average SINR in cases III $\sim$ V.

The SINR of a Rayleigh fading user is an exponential distribution. The mean and variance of $R$ (in $\mathrm{bps} / \mathrm{Hz}$ ) over a Rayleigh fading channel are then given by

$$
\begin{gathered}
E[R]=\int_{0}^{\infty} \log _{2}(1+\overline{\operatorname{SINR}} \times \lambda) \times e^{-\lambda} d \lambda \\
\sigma_{R}^{2}=\int_{0}^{\infty}\left(\log _{2}(1+\overline{\operatorname{SINR}} \times \lambda)\right)^{2} \times e^{-\lambda} d \lambda-(E[R])^{2}
\end{gathered}
$$

where $\overline{\text { SINR }}$ is the average received SINR.

For a $t \times r$ MIMO link over Rayleigh fading channel, where $t$ and $r$ are the numbers of transmit and receive antennas, respectively, there are $m=\min (t, r)$ nonnegative singular values of the channel matrix, each with the same $p d f[13]$

$$
p(\lambda)=\frac{1}{m} \sum_{i=0}^{m-1} \frac{i ! \lambda^{n-m} e^{-\lambda}}{(i+n-m) !}\left[L_{i}^{n-m}(\lambda)\right]^{2}
$$

where $n=\max (t, r), L_{k}^{n-m}($.$) is generalized Laguerre polynomials$ of order $k$. We also assume that the channel information is not known at a sender side, i.e., sender allocates its power equally to all the transmit antennas. In such cases, the mean and variance of a MIMO link over a Rayleigh fading channel are given by [13] [14]

$$
\begin{gathered}
E[R]=\int_{0}^{\infty} \omega(\lambda) \sum_{k=0}^{m-1} \frac{k ! \lambda^{n-m} e^{-\lambda}}{(k+n-m) !}\left[L_{k}^{n-m}(\lambda)\right]^{2} d \lambda \\
\sigma_{R}^{2}=m \int_{0}^{\infty} \omega^{2}(\lambda) p(\lambda) d \lambda-\sum_{i=0}^{m-1} \sum_{j=0}^{m-1}\left[\frac{i ! j !}{(i+n-m) !(j+n-m) !}\right] \\
\times\left(\int_{0}^{\infty} \lambda^{n-m} e^{-\lambda} L_{i}^{n-m}(\lambda) L_{j}^{n-m}(\lambda) \omega(\lambda) d \lambda\right)^{2}
\end{gathered}
$$

where $\omega(\lambda)=\log _{2}(1+\overline{\operatorname{SINR}} \times \lambda / t), \overline{\operatorname{SINR}}$ is the average SINR.

\footnotetext{
${ }^{* 3}$ The SINR in a Rician fading area is typically larger than that in a Rayleigh fading area since there is a dominate component in Rician fading case.
} 
In a Rician fading environment, the SINR of a user is a noncentral chi-square distribution ${ }^{* 1}$ [15] with two degrees of freedom and noncentrality parameter $v^{2}$, where $v$ is the ratio of signal strength in dominant component over the scattered one, so the mean and variance of $R$ over a Rician fading channel are given by

$$
\left\{\begin{array}{l}
E[R]=\int_{0}^{\infty} \log _{2}\left(1+\frac{\overline{S I N R} \times \lambda}{2+v^{2}}\right) \times \frac{e^{-0.5\left(\lambda+v^{2}\right)}}{2} I_{0}(v \sqrt{x}) d \lambda \\
\sigma_{R}^{2}=\int_{0}^{\infty}\left(\log _{2}\left(1+\frac{\overline{\operatorname{SINR}} \times \lambda}{2+v^{2}}\right)\right)^{2} \times \frac{e^{-0.5\left(\lambda+v^{2}\right)}}{2} I_{0}(v \sqrt{x}) d \lambda-(E[R])^{2}
\end{array}\right.
$$

where $I_{0}($.$) is the modified Bessel function of the first kind.$

In the simulation, we set noncentrality parameter $v^{2}=25$ for case IV.

The validities of our theoretical results are illustrated in Figure 1 5. In these experiments, we make several important observations:

(1). the PFS algorithm is bandwidth-efficient. Figure 1 shows that it achieves a spectral efficiency of about $6 \sim 7 \mathrm{bps} / \mathrm{Hz}$ in networks where the highest possible data rate for a user is $8 \mathrm{bps} / \mathrm{Hz}$;

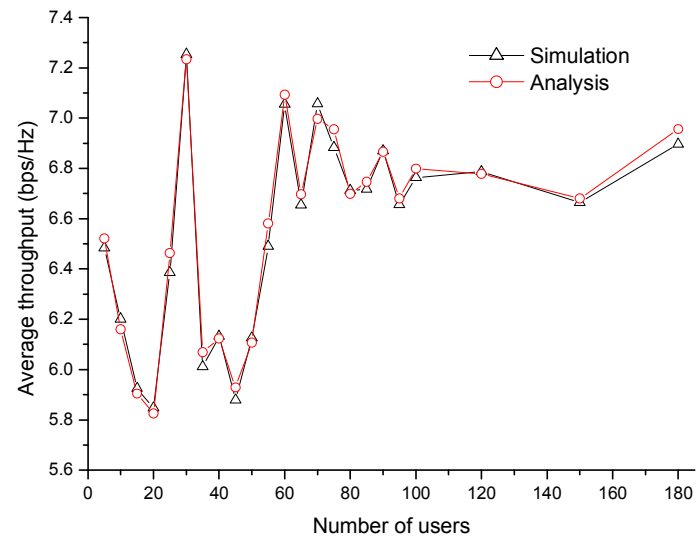

Figure 1. Average overall throughput vs user number: Case I (Gaussian instantaneous data rate with random mean and variance)

(2). Figure 2-5 suggest, while most existing prior results are for Rayleigh fading case, our analytical results apply to Rayleigh, Rician and hybrid fading scenarios;

(3). While existing results only apply to low SINR region (linear rate model) or SISO cases (logarithm rate model), Figure 2-5 show that our analysis apply to both SISO and MIMO networks at low and/or high SINR;

(4). Not surprisingly, Figure 3 indicates a higher average throughput for a Rician fading user as it has dominant component which results in higher SINR than a Rayleigh fading user. Nevertheless, it appears from Figure 4 that the throughput gain of $P F S$ over $R R$ scheduling (i.e., $N \times E[\mu] / E[R]$, where $N$ is the number of users in the network) under a Rayleigh fading

\footnotetext{
${ }^{* 1}$ The mean of a noncentral chi-square distribution with 2 degree of freedom and noncentrality parameter $v^{2}$ is $2+v^{2}$.
}

channel is typically higher than that under a Rician fading channel. From Figure 3, it is fair to predict that users with more fading will get more benefits with SINR being equal.

(5). Figure 4 and 5 show that, for the $P F S$ algorithm in all three fading cases, $E[\mu] / E[R]$ is a monotonically decreasing function of $E[R]$, while $E[\mu] / \sigma_{R}$ is a monotonically increasing function of $\sigma_{R}$. (From (9)-(14), it is easy to prove that $\sigma_{R}$ is a monotonically increasing function of $E[R]$ in Rayleigh/Rician fading environments);

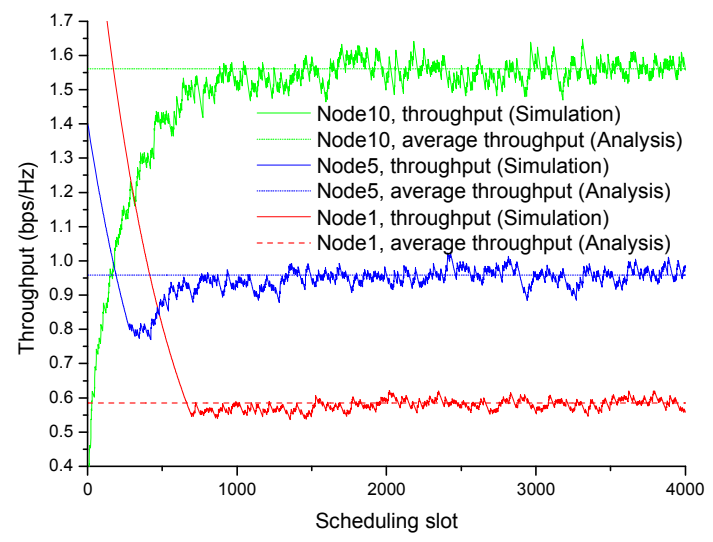

Figure 2. Average overall throughput vs user number Case II ( $5 \times 5$ MIMO, Rayleigh fading)

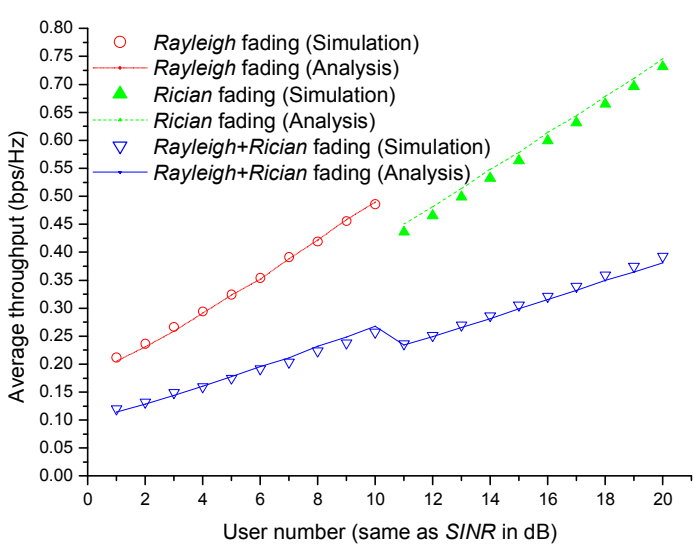

Figure 3. Average throughput of each user Cases III, IV and V (Rayleigh, Rician and Hybrid fading)

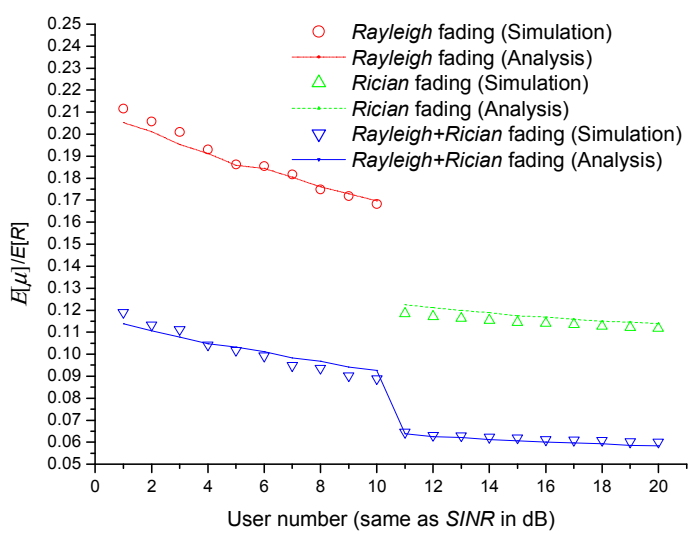

Figure 4. $E[\mu] / E[R]$ : monotonically decreasing with respect to $E[R]$ : Cases III, IV and V (Rayleigh, Rician and Hybrid fading) 


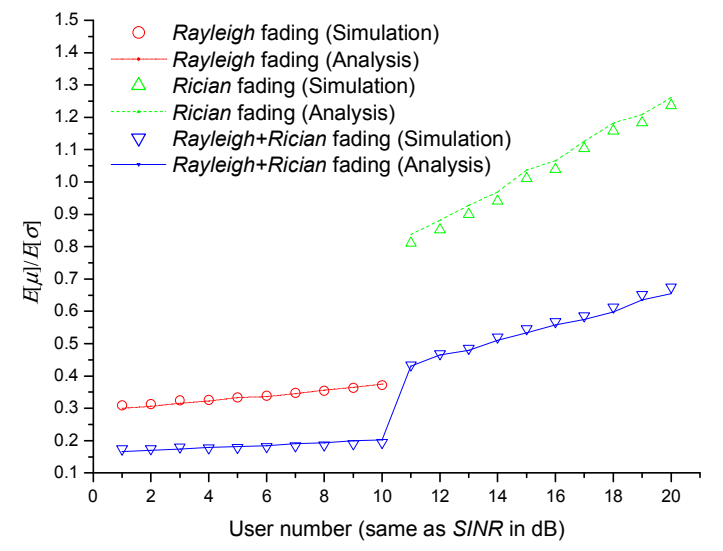

Figure 5. $E[\mu] / \sigma_{R}$ : monotonically increasing with respect to $\sigma_{R}$ Cases III, IV and V (Rayleigh, Rician and Hybrid fading)

The overall conclusion from these experiments is that the closed-form expressions presented in Section III can be used with confidence to evaluate the performance of the PFS algorithm under general fading environments.

\section{CONCLUSIONS}

We have derived a theoretical framework to facilitate researches on PFS. Our contributions are summarized as follows.

- We introduce a new framework to analyze PFS. It has the great practical and theoretical interests of being mathematically graceful and simple. The analytic framework provides very clear physical meaning of the PFS algorithm.

- New exact expressions of the PFS throughput are developed. Simulations show that our formulas remain valid for general (i.e., Rayleigh, Rician and hybrid) fading scenarios. This is in contrast to existing researches that assume Rayleigh fading.

- While existing analytic work are developed for SISO case, the proposed analytic framework provides an extension to analyze PFS in MIMO network.

- Our contribution generalizes existing analytical results by removing the i.i.d restriction used in literature.

Our analysis provides guideline and theoretical support on system design, simulation-based modeling and performance analysis of the PFS algorithm in the context of cross-layer design. The results are being integrated into the MEMBRANE project [16]. For the analysis to be more applicable, we are considering the extension to wireless mesh networks and also its extension to Nakagami fading as a general fading distribution.

\section{ACKNOWLEDGMENT}

This research was financially supported by grants from EU IST FP6 MEMBRANE project under the contract 027310.

\section{REFERENCES}

[1] F. Kelly, "Charging and Rate Control for Elastic Traffic", Eur. Trans. on Telecommun., February 1997, pp. 33-37.

[2] T. D. Nguye and Y. Han, "A Proportional Fairness Algorithm with QoS Provision in Downlink OFDMA Systems," IEEE Commun Lett., vol. 10, no. 11, November 2006.

[3] Z. Han, Z. Ji and K. J. Ray Liu, "Fair Multiuser Channel Allocation for OFDMA Networks Using Nash Bargaining Solutions and Coalitions," IEEE Trans. Commun., vol. 53, no. 8, pp. 1366-1376, August 2005.

[4] A. Jalali, R. Padovani and R. Pankai, "Data Throughput of CDMAHDR: a High Efficiency-High Data Rate Personal Communication Wireless System," in Proc. Veh. Technol. Conf., January. 2001, pp. 5567.

[5] J. M. Holtzman, "Asymptotic analysis of proportional fair algorithm," in Proc. IEEE PIMRC, San Diego, CA, 2001, pp. 33-37.

[6] D. Avidor, S. Mukherjee, J. Ling and C. Papadias, "On Some Properties of the Proportional Fair Scheduling Policy," in Proc. IEEE PIMRC, New Jersey, September 2004, pp. 853-858.

[7] H. J. Kushner and P. A. Whiting, "Asymptotic Properties of Proportional-Fair Sharing Algorithms: Extensions of the Algorithm," in Proc. of the Annual Allerton Conference on Communication, Control and Computing, vol. 41, 2003, pp. 303-311.

[8] J-G. Choi and S. Bahk, "Cell-Throughput Analysis of the Proportional Fair Scheduler in the Single-Cell Environment," IEEE Trans. Veh. Technol., vol. 56, no. 2, pp. 766-778, March 2007.

[9] G. Caire, R. Muller and R. Knopp, "Hard Fairness Versus Proportional Fairness in Wireless Communications: The Single-Cell Case," IEEE Trans. Inf. Theory., vol. 53, no. 4, pp. 1366-1385, April 2007.

[10] M. Andrews, "Instability of the Proportional Fair Scheduling Algorithm for HDR," IEEE Transactions on Wireless Communications, Vol.3, No.5, September 2004.

[11] S. Bost, "User-level performance of channel-aware scheduling algorithms in wireless data networks," in Proc. INFOCOM, San Francisco, 2003, pp. 321-331.

[12] E. Liu and Kin K. Leung, "Proportional Fair Scheduling: Analytical Insight under Rayleigh Fading Environment," in Proc. IEEE WCNC, Las Vegas, March/April 2008.

[13] I. E. Telatar, "Capacity of multi-antenna Gaussian channels," European Transactions on Telecommunications, Vol.10, No.6, pp.585-595, November/December 1999.

[14] P. J. Smith and M. Shafi, "On a Gaussian Approximation to the Capacity of Wireless MIMO Systems," in Proc. IEEE ICC, New York, April 2002, pp. 406-410.

[15] A. Stuart and J. K. Ord, Kendall's Advanced Theory of Statistics, Vol. 2A: Classical Inference \& the Linear Model, 6th ed. New York: Oxford University Press, p. 865, 1999.

[16] The MEMBRANE website. [Online]. Available: http://www.imperial.ac.uk/MEMBRANE 